\title{
A STURM THEOREM FOR PARTIAL DIFFERENTIAL EQUATIONS OF MIXED TYPE
}

\author{
KURT KREITH
}

\begin{abstract}
A generalization of the Sturm comparison theorem is given for differential equations of mixed type. The results constitute a generalization of Sturmian theorems used in the study of hyperbolic initial boundary value problems.
\end{abstract}

Much of the oscillation theory for partial differential equations is based on generalizations of the Sturm comparison theorem to equations of elliptic and hyperbolic types. In the case of the second order elliptic equations there exists an analogue of the Sturm-Picone theorem which leads to a very satisfactory comparison and oscillation theory [3]. However in the hyperbolic case, one seems to be limited to an analogue of the original Sturm theorem, only allowing for the comparison of equations with the same second order terms [1]. This fact constitutes one of the principal barriers to a more satisfactory oscillation theory for hyperbolic equations [4].

The present note is based on the observation that while the Sturmian comparison theorem for second order hyperbolic equations is less general than one would like, it does have one significant advantage: it can be generalized to second order partial differential equations independent of their classifications as hyperbolic, parabolic, or elliptic. Such a generalization enables one to establish oscillation properties associated with certain problems of Tricomi type, as well as providing new insights into hyperbolic oscillation theory.

We shall consider two partial differential equations of the form

$$
\begin{aligned}
& -D_{n+1}\left(m(\mathbf{x}) D_{n+1} u\right)+\sum_{i j=1}^{n} D_{i}\left(h(\mathbf{x}) a_{i j}(\mathbf{x}) D_{j} u\right)+p(\mathbf{x}) u=0, \\
& -D_{n+1}\left(m(\mathbf{x}) D_{n+1} v\right)+\sum_{i j=1}^{n} D_{i}\left(h(\mathbf{x}) a_{i j}(\mathbf{x}) D_{j} v\right)+P(\mathbf{x}) v=0
\end{aligned}
$$

where $\mathbf{x}=\left(x_{1}, \ldots, x_{n+1}\right)$ and the coefficients $m, h, a_{i j}$ are of class $C^{1}$ in the closure of sufficiently regular bounded domain $G \subset \mathbf{R}^{n+1}$ while $p(x)$ and $P(x)$ are assumed to be continuous in $\bar{G}$. It is also assumed that $m(\mathbf{x})$ is positive, while the matrix $\left(a_{i j}\right)$ is symmetric and positive definite in $\bar{G}$. Then the classification of (1) and (2) is completely determined by the sign of the function $h(\mathbf{x})$ for every $x \in \bar{G}$.

Received by the editors January 8, 1980.

AMS (MOS) subject classifications (1970). Primary 35B05.

Key words and phrases. Hyperbolic equations, elliptic equation, nodal domain, characteristic cone. 
For solutions $u(\mathbf{x})$ and $v(\mathbf{x})$ of (1) and (2), respectively, we use the symmetry of $\left(a_{i j}\right)$ to write

$$
\begin{aligned}
\iint_{G} p u v d \mathbf{x} & =\iint_{G}\left[v D_{n+1}\left(m D_{n+1} u\right)-v \sum_{i j=1}^{n} D_{i}\left(h a_{i j} D_{j} u\right)\right] d \mathbf{x} \\
= & \iint_{G}\left\{D_{n+1}\left(v m D_{n+1} u-u m D_{n+1} v\right)-\sum_{i, j=1}^{n} D_{i}\left[h a_{i j}\left(v D_{j} u-u D_{j} v\right)\right]\right\} d \mathbf{x} \\
& +\iint_{G} P u v d \mathbf{x} .
\end{aligned}
$$

Assuming $u=0$ on $\Gamma \equiv \partial G$ and applying the divergence theorem yields

$$
\iint_{G}(p-P) u v d \mathbf{x}=-\int_{\Gamma} v\left(\mathbf{N} \cdot \nabla u-m v_{n+1} D_{n+1} u\right) d s
$$

where $\nabla u=\left(D_{1} u, \ldots, D_{n} u\right), \nu=\left(\nu_{1}, \ldots, \nu_{n+1}\right)$ is the exterior unit normal to $\Gamma$, and

$$
N_{j}=\sum_{i=1}^{n} h a_{i j} \nu_{j} \text { for } 1<j<n .
$$

It clearly follows from (3) that the conditions

$$
\begin{array}{lll}
\text { (i) } & u(\mathbf{x})>0 & \text { in } G, \\
\text { (ii) } & v(\mathbf{x})>0 & \text { in } G, \\
\text { (iii) } & p(\mathbf{x})>P(\mathbf{x}) & \text { in } G \text { and } p \not z P,
\end{array}
$$

are incompatible with the additional hypothesis

$$
\int_{\Gamma} v\left(\mathbf{N} \cdot \nabla u-m v_{n+1} D_{n+1} u\right) d s>0 .
$$

Making use of the linearity of (1) and (2), we therefore have the following result.

TheOREM 1. Let $G$ be a bounded nodal domain for a solution $u(x)$ of (1) and suppose that $p(\mathbf{x}) \geqslant P(\mathbf{x})$ in $G$ with $p \not \equiv$. If (5) is satisfied on $\Gamma$, then every solution $v(\mathbf{x})$ of (2) has a zero in $G$.

An obvious way of satisfying (5) is to assume that $v(x)=0$ on $\Gamma$. However the interest of Theorem 1 derives from the interpretation of the condition

$$
\mathrm{N} \cdot \nabla u-m v_{n+1} D_{n+1} u>0 \text { on } \Gamma
$$

which, together with the assumption $v(x)>0$ in $G$, also suffices to establish Theorem 1.

Writing (6) in the form

$$
\left(\nabla u, D_{n+1} u\right) \cdot\left(\mathbf{N},-m v_{n+1}\right)>0 \text { on } \Gamma
$$

we note that $\left(\nabla u, D_{n+1} u\right)$ is in the direction of the interior normal to $\Gamma$. In the elliptic case $h(\mathbf{x})<0$, the second vector $\left(\sum_{i=1}^{n} h a_{i 1} \nu_{1}, \ldots, \sum_{i=1}^{n} h a_{i n} \nu_{n},-m \nu_{n+1}\right)$ is also directed interior to $\Gamma$. As a result, in the elliptic/parabolic cases (where $h(\mathbf{x})<0),(5)$ is always satisfied without requiring that $v(\mathbf{x})$ vanish on $\Gamma$. 
In the hyperbolic case $(h(x)>0),(6)$ may not be satisfied on $\Gamma$. However there is a simple and geometrically interesting way of decomposing $\Gamma$ into

$$
\Gamma_{1}=\{\mathbf{x} \mid(6) \text { is satisfied at } \mathbf{x}\}, \quad \Gamma_{2}=\{\mathbf{x} \mid(6) \text { is not satisfied at } \mathbf{x}\} .
$$

In this connection we choose an arbitrary $x_{0} \in \Gamma$ and make a canonical change of variables for which $h\left(\mathbf{x}_{0}\right)=1, m\left(\mathbf{x}_{0}\right)=1$, and $a_{i j}\left(\mathbf{x}_{0}\right)=\delta_{i j}$. Assuming $G$ to be a nodal domain for a solution $u(\mathbf{x})$ of (1) satisfying $u(\mathbf{x})>0$ in $G$, it again follows that $\left(\nabla u, D_{n+1} u\right)\left(\mathrm{x}_{0}\right)$ is an interior normal to $\Gamma$ while

$$
\begin{aligned}
\mathrm{t} & =\left(\nu_{1}, \ldots, \nu_{n},-\frac{\nu_{1}^{2}+\cdots+\nu_{n}^{2}}{\nu_{n+1}}\right) \quad\left(\nu_{n+1} \neq 0\right) \\
& =\left(\nu_{1}, \ldots, \nu_{n}, 0\right) \quad\left(\nu_{n+1}=0\right)
\end{aligned}
$$

is the tangent to $\Gamma$ at $\mathbf{x}_{0}$ with projection $\left(\nu_{1}, \cdots, \nu_{n}\right)$ into $\mathbf{R}^{n}$. Also, the interior of the characteristic cone associated with (1) and (2) at $\mathbf{x}_{0}$ is generated by vectors $\xi=\left(\xi_{1}, \ldots, \xi_{n+1}\right)$ originating at $x_{0}$ and satisfying $\xi_{1}^{2}+\cdots+\xi_{n}^{2}<\xi_{n+1}^{2}$. Now at $\mathbf{x}=\mathbf{x}_{0}$, (7) reduces to $(-\nu) \cdot\left(\nu_{1}, \ldots, \nu_{n},-\nu_{n+1}\right)>0$ or $\nu_{1}^{2}+\cdots+\nu_{n}^{2}<\nu_{n+1}^{2}$, which is precisely the condition that the tangent vector $\vec{t}$ at $x_{0}$ not be interior to the characteristic cone. Denoting the interior of the characteristic cone at $\mathbf{x}$ by $\mathcal{C}(\mathbf{x})$, we have

$$
\begin{aligned}
& \Gamma_{1}=\{\mathbf{x} \in \Gamma \mid \text { the tangent to } \Gamma \text { at } \mathbf{x} \text { does not belong to } \mathcal{C}(\mathbf{x})\}, \\
& \Gamma_{2}=\{\mathbf{x} \in \Gamma \mid \text { the tangent to } \Gamma \text { at } \mathbf{x} \text { belongs to } \mathcal{C}(\mathbf{x})\}
\end{aligned}
$$

With these definitions, Theorem 1 takes on a new meaning.

Theorem 2. Let $G$ be a bounded nodal domain for a solution $u(x)$ of (1) and suppose that $p(x) \geqslant P(x)$ in $G$ with $p \neq P$. If $v(x)$ is a solution of (2) satisfying $v(\mathbf{x})=0$ for all $x \in \Gamma_{2}$, then $v(\mathbf{x})$ has a zero in $G$.

By way of examples, we note that in [1], a Sturmian comparison theorem was established (in different notation) for hyperbolic initial boundary value problems of the form

$$
-\left(m(y) u_{y}\right)_{y}+\left(h(y) u_{x}\right)_{x}+p(y) u=0 ; \quad 0<x<\pi \text { and } y>0,
$$

satisfying $u(0, y)=u(\pi, y)=u(x, 0)=0$. Separating variables by writing $u(x, y)$ $=Y(y) \sin x$ yields

$$
-\left(m Y^{\prime}\right)^{\prime}+(p(y)-h(y)) Y=0 ; \quad Y(0)=0,
$$

which is oscillatory if $p(y)-h(y)$ is sufficiently negative. Assuming $Y(y)$ has a zero $y_{1}>0$, the above separation of variables yields a nodal domain $G=\{(x, y) \mid 0$ $\left.<x<\pi, 0<y<y_{1}\right\}$ to which Theorems 1 and 2 can be applied. Since the segments $(0<x<\pi, y=0)$ and $\left(0<x<\pi, y=y_{1}\right)$ clearly belong to $\Gamma_{1}$, we have the following result reminiscent of [1]: If $P(x, y)<p(y)(P \not \equiv)$ and $v(x, y)$ is a solution of

$$
-\left(m(y) v_{y}\right)_{y}+\left(h(y) v_{x}\right)_{x}+P(x, y) v=0
$$

satisfying $v(0, y)=v(\pi, y)=0$ for $0<y<y_{1}$, then $v(x, y)$ has a zero in $G$. However, Theorem 2 allows one to improve on this conclusion in case $h(y)$ 
becomes negative. In particular $\{y \mid h(y)<0\}$ generates more boundary points belonging to $\Gamma_{1}$ and thereby enlarges the class of solutions of (2) to which the conclusion of Theorem 2 applies. For example, if we consider the Tricomi equation (2') with $h(y)<0$ for $0<y<\tilde{y}\left(\tilde{y}<y_{1}\right)$ and $h(y)>0$ for $\tilde{y}<y<y_{1}$, then $v(x, y)$ need only satisfy $v(0, y)=v(\pi, y)=0$ for $\tilde{y}<y<y_{1}$ in order for Theorem 2 to apply.

For the equation

$$
-u_{y y}+u_{x x}+p(x, y) u=0
$$

it is especially easy to determine $\Gamma_{1}$ and $\Gamma_{2}$. If $G$ is a nodal domain for a solution of ( $\left.1^{\prime \prime}\right)$ and $\Gamma$ is given (locally) as the graph of $y=f(x)$, then

$$
\begin{aligned}
& \left(x_{0}, f\left(x_{0}\right)\right) \in \Gamma_{1} \quad \text { if and only if }\left|f^{\prime}\left(x_{0}\right)\right|<1, \\
& \left(x_{0}, f\left(x_{0}\right)\right) \in \Gamma_{2} \quad \text { if and only if }\left|f^{\prime}\left(x_{0}\right)\right|>1 .
\end{aligned}
$$

Thus the fact that $u(x, y)=a^{2}-x^{2}-y^{2}$ satisfies $-u_{y y}+u_{x x}=0$ and has a circular nodal domain, $G=\left\{(x, y) \mid x^{2}+y^{2}<a^{2}\right\}$, implies that if $P(x, y)<0$ in $G$, then every solution of

$$
-v_{y y}+v_{x x}+P(x, y) v=0
$$

which is zero on $\Gamma_{2}=\left\{(x, y) \in \Gamma \mid y^{2}<x^{2}\right\}$ also has a zero in $G$.

As a final example, we note that solutions of characteristic initial value problems of the form [2]

$$
\begin{array}{cc}
u_{s t}+p(s, t) u=0 ; & s>0, t>0, \\
u(0, t)=\varphi(t) ; & u(s, 0)=\psi(s)
\end{array}
$$

frequently have nodal lines which are the graphs of $t=f(s)$ where $f^{\prime}(s)<0$ for $0<s<\infty$. The question arises as to whether two such nodal lines could intersect twice to enclose a nodal domain $G$ for a solution of (8).

A negative answer can be given by noting that such a nodal domain would have $\Gamma=\Gamma_{1}$ and $\Gamma_{2}=\varnothing$. Choosing a constant $P<p(s, t)$ in $G$, one can always choose initial values $\Phi$ and $\Psi$ so that the solutions of

$$
v_{s t}+P v=0, \quad v(0, t)=\Phi(t), \quad v(s, 0)=\Psi(s)
$$

has no zeros in $G$, and the existence of this solution would contradict Theorem 2.

\section{REFERENCES}

1. K. Kreith, Sturmian theorems for hyperbolic equations, Proc. Amer. Math. Soc. 22 (1969), $277-281$.

2. G. Pagan, Oscillation theorems for characteristic initial value problems for linear hyperbolic equations, Accad. Naz. Lincei 55 (1973), 301-313.

3. C. A. Swanson, Comparison and oscillation theory of linear differential equations, Academic Press, New York, 1968.

4. __ A dichotomy of PDE Sturmian theory, SIAM Rev. 20 (1978), 285-300.

Department of Mathematics, University of California, Davis, California 95616 